\title{
Obreras y empresarias en el Periodo Romano Alto Imperial
}

\author{
Workers and entrepreneurs in the High Roman Empire
}

\author{
Pilar Fernández URIEL* \\ A mi amigo FEDERICO LARA PEINADO, \\ catedrático de Historia Antigua
}

\begin{abstract}
RESUMEN ABSTRACT
Análisis de la situación laboral y las diferentes medios y trabajos de las mujeres en el contexto de la Roma alto Imperial, frente a la situación mentalidad tradicional femenina a través de la documentación que proporcionan las fuentes históricas.

Se contempla su relación con la sociedad, su ámbito doméstico e incluso la posible repercusión que pudiera existir en la cultura y mentalidad de este momento histórico.

Analysis of the labor situation and the different ways and works of women in the context of high Imperial Rome, facing the situation traditionally minded women through the documentation provided by historical sources. It sees its relationship with society, its domestic and even the possible impact that might exist in the culture and mentality of this historical moment.

\section{PALABRAS CLAVE:}

KEYWORDS:
\end{abstract}

Inscripción, mujer, negotiatrix, officina, propietaria, administradoras, comercio.

Inscription, woman, negotiatrix, officina, owners, managers, trade.

\section{INTRODUCCIÓN}

La inscripción sepulcral de la matrona romana, Claudia, dice así:

Hospes, quod deico paullum est; asta ac pellege.

Heic est sepulcrum hau pulcrum pulcrae feminae; nomen parentes nominarum Claudia.

* Departamento de Historia antigua. UNED. e-mail: pfuriel@geo.uned.es 
Suom mareitum corde deilexit souo:

gnatos duos creauit: horunc alterum

in terra linquit, alium sub terra locat.

Sermone lepido, tum autem incessu commodo.

domum semauit. lanam fecit. dixi. abei. (CIL, VI, 3.15346;. POMEROY, S., 222).

«Forastero, lo que tengo que decir es corto. Párate y lee esto.

Esta es la tumba de una hermosa mujer. Sus padres y su marido la llamaban Claudia. Amaba a su marido con todo su corazón. Tuvo dos hijos, a uno de los cuales lo dejó en la tierra, al otro lo dejó bajo la tierra. Fue encantadora en su conversación y su conducta fue la apropiada. Cuidó de su casa e hilaba la lana. Puedes irte"(Trad.propia).

El epitafio de Amymone expresa las virtudes de la difunta en términos muy semejantes:

Hic sita est Amymone Marci optima et pulcherrima, lanifica, pia, pudica, frugi, casta, domiseda (CIL VI, 11602; ILS 8402).

«Aquí reposa Amymone, esposa de Marco, ejemplar y bellísima mujer, que hilaba la lana, pía, púdica, honesta, casta, dedicada al cuidado de su hogar» (Trad. propia).

Estos epitafios funerarios describen como debía comportarse en la vida de una mujer tradicional y lo que se exigía y esperaba de ella en la Roma del final de la República y del Alto Imperio, con adjetivos que se repiten (casta, pia, frugi, domiseda, lanifica).La mujer debía ser abnegada en el hogar, capaz de someterse y sacrificarse. De hecho, Columella considera la mujer inhábil para otro trabajo que no fuera el doméstico y a tender la casa (COLUMELA De agric., Prefacio, L. 12). (CID LÓPEZ, R. M., 2001, 19-44; CANTARELLA, E., 1991, 96).

Pero no todas las mujeres limitaron sus tareas a hilar y cuidar de su casa, como se esperaba de ellas. Otras ejercieron diversas profesiones, impulsadas por motivos tan diversos como su situación social, sus medios económicos, su preparación, su ingenio e, incluso, su valor.

Son muy escasos los testimonios que informan sobre la actividad laboral de la mujer romana, excepto algunas alusiones en las fuentes literarias que hay que interpretar con mucho prudencia, en especial la sátira de Marcial y Juvenal, la mayor parte de los testimonios proceden de la epigrafía, y más concretamente de los epitafios funerarios, teniendo en cuenta la limitación de datos que aportan ya que el testimonio que nos han llegado suele reducirse a unas breves líneas alusivas a su vida. La iconografía es una valiosa fuente documental y en muchas ocasiones, enormemente elocuente (LE GALL, J.1969, 123-130).

A pesar de la parquedad de las fuentes históricas, el abanico de trabajos y oficiosa los que se dedicaron estas mujeres abarca un amplio espectro, son muy di- 
versos y diferentes, existiendo notables diferencias tanto en su capacidad económica como en reconocimiento y status social (ALFARO, C. (ed.), 1999, 205-359).

\section{MULIERES NEGOTIATRICES}

El término Negotiatrix es difícil de definir y determinar. Puede referirse simplemente a las actividades comerciales o ampliar este concepto a toda clase de acciones relacionadas con el ingreso y especulación del dinero.

Ateniéndonos al concepto latino (femenino de negotiator) se refiere simplemente a la mujer que mercadea, diferenciándose de la que se dedica a otra clase de ocupación.

Durante el Alto Imperio el término se refiere a la mujer que ejerce todo tipo de prácticas especulativas, por lo general este término suele ir acompañado por el nombre del ámbito comercial y especifica el tipo de mercado al que se dedica.

M. Eichenauer va más lejos concretando más esta definición y considera que el término se vincula muy especialmente al comercio del vino, el aceite y sobre todo, a los cereales (EICHENAUER, M., 1988, 23).

Sin embargo, el término negotiator aparece con frecuencia vinculado a otro tipo de operación empresaria como la fábrica de tejas y materiales de construcción.

Respetando la terminología latina, seccionaré las dedicaciones de estas «mujeres empresarias» entre las dedicadas a la banca y la especulación monetaria y las relacionadas con la actividad mercantil, diferenciando también su consideración social y económica.

\section{ALTA CLASE SOCIAL: MUJERES EMPRESARIAS}

\section{PRÉSTAMOS Y CONTRATOS. LA BANQUERA CESENIA}

Caesenia era una patricia. Tanto ella como su esposo, el banquero Marco Fulcino, descienden de ricas y poderosas familias de la aristocracia de Tarquinia. Al morir su marido y su hijo, Caesenia heredó todos los bienes, y además quedó libre de la patria potestad de ambos (tutela murieris) y pudo disponer libre de este patrimonio (SALLER, R., 1999, 84-87).

Debía gestionar sus bienes y resolver los asuntos que quedaban pendientes al morir su marido, para lo que eligió como administrador al liberto Ebucio. No hizo buena lección y Ebucio intentó engañarla.

Conocemos las gestiones de Caesenia a través de la obra de Cicerón ( $D e$ fensa de Aulo Cecina IV, 6, 10), donde es alabada como digna y honradísima romana sin menoscabar su honestidad y reputación propias de una mujer perteneciente al patriciado romano. Considera procedente que administre su patrimonio y ejerza en las actividades exigidas para gestionar sus fincas, defenderse en litigios 
y saber desenvolverse y relacionarse con los banqueros de Roma (GARCIA GARRIDO, M. J., 2001, 69; ANDREAU, J., 2001)1.

Otras damas no salen tan bien paradas, como Octacilia Laterense que, según Valerio Máximo, quiso engañar a los herederos de su amante Cayo Viselio Varrón cuando este cayó gravemente enfermo, exigiendo la considerable cantidad de 300.000 sestercios a través de un contrato anteriormente realizado entre ambos (trascriptium nominum), pero Varrón se recuperó y Octacilia fue castigada duramente por el juez Cayo Aquilio Galo. Sin duda, Octalicia Laterense no parecía adecuarse a los valores y comportamiento que la sociedad romana de Valerio Máximo consideraba propios de una mujer, no cumplía con los deberes que se esperaba de ella y fue acusada de adúltera e inexorable usurera.

"Cayo Viselio "Varrón, atacado por una peligrosa enfermedad, consintió en reconocerse deudor por una suma de 300.000 sestercios de Otalicia Laterense, con la que había tenido relaciones amorosas". Su intención era que si se moría, ella podía reclamar dicha suma a los herederos. Se trataba, pues, de un legado testamentario por el que quería encubrir su inmoral liberalidad, bajo el nombre de crédito.

Pero contra los deseos de Otalicia, Viselio curó de su enfermedad. Irritada ésta por no haber podido apropiarse enseguida de lo que esperaba con la muerte del enfermo, abandonó el papel de amiga complaciente y se convirtió, de repente, en una inexorable usurera. Exigió el pago de aquella suma de la que había tratado de apoderarse por medio de una actitud desvergonzada y de un contrato sin valor.

Fue Ilamado como juez a Cayo Aquilio Galo, hombre de gran prestigio y muy versado en derecho civil. Este, después de consultar a los ciudadanos más influyentes de la ciudad, rechazó las pretensiones de la mujer con prudente sabiduría.

El juez castigó personalmente la intriga de una acción privada, pero dejó a los tribunales públicos el cuidado de investigar el adulterio" NALERIO MAXIMO, Fact. Men, VIII, 2,2)2.

Hay otros testimonios sobre mujeres que llevaron a cabo transacciones comerciales, préstamos y usura.

Muchas de estas mujeres (posiblemente iletradas), aun poseyendo el iustrium que permitía la gestión propia de sus bienes (sin la interferencia del marido o del padre), pudiendo legalmente heredar y legar. La actividad comercial e inversora proporcionó a las mujeres la posibilidad de actuar e invertir a través de terceros, plenamente acorde con la ideología que imperaba en cuanto a la conceptualización de la virtud femenina. Ellas se valían de tutores para realizar transacciones, firmar documentos y asegurar la legitimidad de las operaciones, en muchas de las cuales

1 Sobre la dedicación de la mujer a los negocios y la Banca la opinión general queda reflejada por el Jurista Calístrato: "Se considera que las mujeres quedan excluida del oficio de banquero, pues es cosa propia de hombres (Digesto, 2.13.12).

2 Se ha utilizado la obra de Valerio Máximo, Trad. de la Biblioteca Clásica Gredos.; También: MARTÍN ACERRA, F. Valerio Máximo. Hechos y dichos memorables, Madrid, 1988; MONTERO, S., 2004, 45-56; PEPPE, L., 1984. 
la ley exigía que fueran representadas por un garante o un tutor que figuraba al lado del nombre de la dama ${ }^{3}$.

Las tablillas halladas en Murécine, una localidad cercana a Pompeya, son unos de los documentos más importantes con los que contamos. Están fechados en torno al año 61 d.C., pertenecen al archivo de la casa de los Sulpicios, una familia banquera de origen liberto.

En el libro de contabilidad de Sulpicio Cinano figura la deuda contraída por una liberta de origen griego Eupliae, hija de Teodoro, interviniendo como garante Epichare Afrodisio y la operación se realiza «Domo», en la propia casa (BOVE L., 1979; CAMODECA, G. 1999).

Otra tablilla cita a la liberta Popea Data, liberta de Prisco, que, al parecer, había logrado alcanzar una cierta solvencia económica con pequeños negocios. Trató con otra mujer Decidia Margaris un préstamode1.450 sestercios, que ha de devolver en un plazo determinado, dejando como garante (actio depositi) dos esclavos.

Hay más préstamos realizados por mujeres como el que Vettia, que consiguió 20 denarios a un interés de 12 ases, o el que realizó Faustilia, 15 denarios al interés de 9 ases, ambos anuales. Es posible que todas estas pequeñas transacciones se debieran a necesidades de tipo doméstico (SALLER, R., 1999,84-87).

Fuera de la península Itálica, los famosos papiros de Oxirrynco en Egipto informan de contratos donde intervienen mujeres (AA.VV. 1998-2007 y1999).

Uno de cierta envergadura fue celebrado en la localidad egipcia de Tebtinis, en el año 140 d.C, concertado entre damas: Dydime prestó la cantidad de 372 dracmas de plata a otras dos mujeres Ilamadas Taorsenousis y Terphosais cantidad que debía restituirse en cinco años, teniendo como garante el usufructo de varias tierras. Ambas partes estuvieron asistidas por su respectivo tutor.

Realmente, hubo una gran diversidad de negocios y formas de ganar dinero realizados por mujeres. En los papiros descubiertos en Egipto hay numerosas referencias a contratos, algunos con intervención de los respectivos tutores:

Un curioso contrato es el de lactancia que realiza Filotera, arrienda o alquila a su esclava Zosime a su propia hija Sillis para que sirva de nodriza y alimente con su leche a la recién nacida esclava Agalmation por un período de dos años con el salario mensual de 12 dracmas de plata establecidas por la leche y la crianza, a cambio Filotera ha recibido aceite, pan y la suma total de 288 dracmas, fechado

3 Las Leyes de Augusto en el 18 y 17 a. C. y en el 9 d. C. dispuso una serie de leyes revolucionaron el derecho de familia y liberaron en gran parte a las mujeres de la sumisión a esta tutela y se proporcionó a la mujer una cierta libertad gracias al llamado iustrium liberorum. Una mujer libre que hubiera tenido tres hijos (casada o no) y una liberta que hubiera tenido cuatro, quedaban libres de tutela. Poco después con Claudio (s. I d. C.) la tutela queda automáticamente abolida para las mujeres libres. GAUDEMET, J, 1959; TREGGIARI, S. 1993; LÁZARO GUILLAMÓN, C., 2003, DEL CASTILLO, A, 1988-89. 
en Alejandría en el año 13 a.C. tiene como tutor al marido y padre respectivo, Ptolemaios.

No son, ciertamente, grandes operaciones, la mayoría de las cantidades no son muy considerables, pero tenemos información de otras Dominas que figuran al frente de empresas de importante calado. Las fuentes también incluyen a mujeres dentro del término Negotiatores, que además aparecen extremadamente especializadas en su actividad durante el periodo imperial.

\section{PROPIETARIAS Y ADMINISTRADORAS}

La mujer no sólo podía ser propietaria de tierras rústicas (Fundi) sino también de bienes inmuebles, gozaban cierta solvencia y estabilidad socio-económica pudiendo vivir de sus rentas. Pero interesan aquí las que, lejos de limitarse al usufructo de sus tierras y bienes inmuebles, sabían explotar y negociar con ellos (CASTILLO, A. del, 1974; LÁZARO GUILLAMÓN, C., 2003).

Se conocen muchos nombres de mujeres propietarias de latifundios (Fundi) pero son muy pocas las que administran y trabajan en ellos. Valeria Maxima era una matrona romana que puso al frente de sus fincas no un capataz (Villicus) sino dos Villicae, dos mujeres que se llamaban Eucrotia y Cania Urbana, ambas citan a su Domina en su epitafio fúnebre, el de esta última dedicado por su hija Lucania Ursina y su esposo Tatio Verecundiano.

D M/ CANIAE VRBANAE/ VILLICAE QVAE/ VIXIT ANN LVCANIA/ VRSINA FILIAE/ TATTIVS VERECVNDIANVS/ GENER/ MATRI INCOMPARABILI (CIL III, 2118)

Eucrotia y Cania Urbana no fueron un caso excepcional, Prastina Maxima era una, intendente (auctrix) lo que comporta la gestión y administración de bienes de una familia senatorial:

B M PRASTINIA MAXIMINA ACT/ RIX C DOMVS QVE IVNCTA EST MARITO/ ANNORVM SECEDIM SIC ET POSSEDIT MARITVM/ ANNIS XVI FILIOS DI/ N TRERES PER (CIL XI, 1730)

La epigrafía informa de otras mujeres que eran propietarias de bienes inmuebles con los que invertían y negociaban realizando compras, ventas y alquileres como la pompeyana Julia Felix (CIL IV, 1136) que alquilaba y vendía locales comerciales: (MAIURI, A., 1983.,75-84; PARSLOW, Ch C., 1989).

El epitafio de Sergia Paulina recoge la referencia a un collegium que tiene su sede en una parte de la casa de esta propietaria.

D M/ HERMEROTI/ ARCADIO V A XXXIV/ COLLEGIVM/ QVOD EST IN DOMV/ SERGIAE PAVLLINAE/ FECERVNT/ AGATHEMER/ ET/ CHRESTE ARESCON/ FRETRI PIISSIMO B M: (CIL VI, 9148 = ILS 7333)

Las fuentes proporcionan nombres de mujeres negotiatrices con una actividad comercial relacionada fundamentalmente con el cereal, pero también con el vino y 
el aceite lo que hacen deducir a Jefferson Loane que, con probabilidad, se trata de mujeres que habrían heredado de padres o maridos este negocio y que les impulsó a continuar con el negocio familiar.

Abundia Megiste fue una «negotiatrix frumentaria et leguminaria ab scala mediana». Compartía el trabajo con su marido, el ciudadano romano M. Abundio Luminaris, quien además era su patrono y que tenía otros libertos. Cuando se quedó viuda continuó dirigiendo ella sola el negocio de compra venta de cereal que conocía como nadie. (CIL VI, 9683 = ILS 748839; LÁZARO GUILLAMÓN, C., 2003).

El comercio del vino proporcionaba grandes beneficios sobre, todo de Hispania ${ }^{4}$.

Las mujeres que se dedicaban al negocio de la compra venta del vino plantean un problema concreto ¿están dedicadas sólo a su mercado o regentan un establecimiento tipo Caupo? Es posible que las que aparecen como Calpurnia en un epitafio tuvieran, además de una cierta solvencia económica, una cierto status social en su trabajo para ser reconocidas como tales en su inscripción funeraria pompeyana:

SVAVIS VINARIA/ SITIT ROGO VOS ET/ VALDE SITIT/ CALPVRNIA TIBI DICIT VAL (CIL IV, 1819)

\section{¿MUJERES NAVIERAS? (NAVICULARII)}

Como manifiesta J. Rougé, la mujer naviera pudo tener una relación directa con la navegación comercial como propietaria o inversora en barcos, los patronos de la nave (magister navis) estaba siempre en dependencia con el propietario (Digesto. $14,1,1,5)$.

En un texto de Suetonio parece clara la participación de la mujer en la industria naval:

«Habiendo encarecido bastante el precio de los víveres a consecuencia de una prolongada escasez, se produjo un grave tumulto contra él en el Foro de Roma y para garantizar el abastecimiento concedió algunas ventajas a quienes equipasen naves para el comercio de granos proporcionadas a su posición en el Estado, otorgó a los ciudadanos las dispensas establecidas por la Ley Papia Popea, a los latinos los derechos de ciudadanos romanos, a las mujeres las prerrogativas de madres de cuatro hijos, subsistiendo aún hoy tales reglamentos» (SUETONIO, Vita Claudio, XIX).

Un periodo de falta de víveres y malas cosechas provocó una revuelta popular durante el principado de Claudio obligándole a conceder determinados beneficiosa

4 EICHENAUER, M, 1988; JEFFERSON LOANE, H., 1990; GALLEGO FRANCO, H.: 1993, pp. 114 y 115 111-127; MORETTA, S., 1999, 229-245, que aporta una interesante relación de nombres femeninos en TituliPicti. Más ejemplos de negotiatrices de vino y aceite pueden hallarse en varias inscripciones (CIL XV, 3691, 3729, 3845-7). 
naviculari y comerciantes e, incluso, a contraerlas pérdidas que éstos pudieran sufrir en sus transacciones y trasporte del cereal.

Entre estos beneficios destaca la concesión de relevantes prerrogativas a los armadores de naves de carga según su condición. Así, a los ciudadanos romanos les eximió de la sanción contra los célibes y matrimonios sin hijos de la Lex Papia Poppaea y la ciudadanía romana a los latinos y a las mujeres les concedió los privilegios de las madres de cuatro hijos (TREGGIARI, S., 1993).

Esta última apreciación permite deducir que hubo mujeres navieras, propietarias de barcos entre los negotiantes dedicados al transporte de la annona a la ciudad de Roma, y probablemente, al menos parte de ellas, eran libertas debido a la referencia al ius liberorum, y en concreto, «los privilegios recibidospor cuatro hijos». García Garrido considera este testimonio una clara muestra de la participación de las mujeres en la industria naval.

La parte final del texto quae constituta hodieque servantur hace suponer su permanencia al menos en el Alto Imperio, y es posible que perdurara en épocas posteriores.

También hay ejemplos de capitalistas o inversoras que J. Rougé y P. Grimal denominan navicularios honorarios, es decir mujeres que exponían el capital en naves y asumen el mismo grado de responsabilidad que sus colegas navieros tal y como se desprende del pasaje de Suetonio (ROUGÉ, J, 1966; GRIMAL, P., 2007).

En este contexto, se supone que hubo mujeres de alta capacidad económica, dado que la inversión pecuniaria que debía realizarse como propietarias de barcos costeando su mantenimiento, podían llegar a ser muy alta y además se encontraban perfectamente amparadas por el ordenamiento jurídico (SETÄLÄ P. et alii, 2002).

Una pujante mujer empresaria fue Domicia Lucila, en torno al 108 d.C., propietaria de unas canteras de arcilla que supo explotar con una fábrica de tejas y materiales de construcción cuyos sellos aportan una interesante información de su empresa, en especial las tejas (CIL XV, 263 y 264). Debió tener una producción grande con esclavos y obreros y otros subordinados, incluso sabemos que tenía dos encargados: uno al frente del trabajo las canteras (servus vicarius) denominado Agotobulo y como encargado de la fábrica (officinator) a un talTrofino.

No debió ser Domicia la única dedicada a este tipo de empresa según F.E Adcock, los nombres de mujeres aparecen frecuentemente en sellos de ladrillos, dado que muchas de ellas eran propietarias de las fincas en las que se ubicaban pozas de arcilla que beneficiaban o arrendaban para su explotación, es más, en alguno de estos sellos aparece la expresión negotiator vinculada a un nombre femenino (ADCOCK, F. E., 2009, 77-100).

Dentro del ámbito de los negocios dedicados a los materiales de construcción, podríamos citar a la liberta Antistia Delphidis, tesseraria lignaria, fabricante de tesserae de madera: 
D M/ ANTISTIAE GAIAE/ L DELPHIDIS/ TESSER LIGNAR/ SODALICI MARMORARIOR. (CIL V, $7044=$ ILS 7288)

Aurelia Vibia Sabina figura como Domina del servus marmorarius Hermes que, posiblemente trabajaba en un taller de su propiedad (CIL II, 133, J. D'ENCARNAÇAO, 1984, no 497).

Cornelia Cruseidisera domina de Agathocules y propietaria del taller donde este trabajaba como inaurator o dorador (GALLEGO FRANCO, H., 1993, 116).

Y como no citar a la pompeyana Eumachia, una respetada mujer empresaria y una de las más influyentes comerciantes de la ciudad. Pertenecía a la familia de los Eumachii propietarios territoriales y poderosos industriales. R. Etienne la define como «mujer de negocios» (ETIENNE, R., 1992, 184 ss.).

Al no haber aún confirmación de mujeres adscritas a gremios comerciales, no es posible incluir a Eumachia dentro del concepto de collegium en sentido estricto, aunque se considera patrona de los bataneros de Pompeya. Era la protectora de los lavanderos de lana, tejedores, tintoreros y blanqueadores, incluso, muy probablemente, por su intensa actividad comercial, también podía estar vinculada al mundo de la navegación como mecanismo de distribución de sus propios productos (CANTARELLA, E., 1999).

Destaca el edificio que construyó para la ciudad en su nombre y en el de su hijo Marco Numistrio Frontón. Posiblemente era el mercado de la lana o la sede del gremio de los fullones. Fue dedicado a la Concordia y a la Piedad Augusta, así como a Livia, esposa de Augusto, según la inscripción del arquitrabe del pórtico de dos pisos, sostenido por columnas, situado en el interior. Este portal está enmarcado por un relieve de mármol de una gran belleza, decorado por pájaros, hojas acanto e insectos, parecidos al Ara Pacis, confirmando la admiración y homenaje de Eumachia por la familia de Augusto 5 .

EVMACHIA L - F - SACERD - PVBL - NOMINE SVO - ET - M - NVMISTRI FRONTONIS.FILI. CHALCIDICVM - CHRYPTAM. PORTICVS CONCORDIAE AVGVSTAE PIETATI. SVA. PEQVNIA - FECIT EADEMQVE - DEDICAVIT. (CIL X, $810=$ ILS. 3785).

La literatura también alude a mujeres empresarias. El ejemplo de liberta astuta y emprendedora es Fortunata, esposa de Trimalción, ambos libertos de reciente fortuna (adquirió todas las tierras de su patrono y entre sus actividades comerciales destacan la de armador de barcos dedicados al comercio de vino, legumbres y manteca de cerdo).

5 ROUGÉ, J., 1966, 274 ss; PAPPALARDO, U. 2002-2003 pp. 171-178; SPANO, G., 1961; CANTARELLA, E. - JACOBELLI, L, 1999, pp. 24-26; COARELLI, F.,- LAROCCA, E., - de VOS, M.1980; GUIRAL PELEGRÍN, C., MOSTALAC CARRILLO, A.: 2008, pp. 59-68; ZANKER, P., 1993; JONGMAN, W. 1991. 
En el conocido capítulo de la cena de Trimalción, el que el anfitrión y su esposa hacen ostentación de su riqueza, así se define a Fortunata:

«Comencé a tirar de la conversación desde muy atrás, preguntándole quién era una mujer que corría de aquí para allá:

La señora de Trimalción -me repuso- y mide su dinero por arrobas. $Y$ hace nada, nada ¿qué era? Con perdón de tu cara, no habrías cogido de su mano ni un trozo de pan. Y ahora, sin cómo ni por qué, se subió a las nubes y es el factotum de Trimalción. Es decir, en pleno mediodía si le dijera que es de noche, él se lo creería. No sabe lo que tiene de lo riquísimo que es; pero ella tiene los ojos en todas partes y se encuentra siempre hasta donde no la esperan. No es bebedora, no es comedora, es muy sentada/ vale lo que pesa, ahora que, en cambio, es deslenguada y chismosa: a quien quiere, quiere; a quien no quiere, no quiere" (Satyr.XXXVII). (ADCOCK, F. E, 1945).

\section{MUJERES PURPURARIAS:}

Está perfectamente constatado que las mujeres trabajaron en un negocio tan lucrativo como era la producción y el comercio de la púrpura.

La inscripción que se encuentra en lápida funeraria fechada a finales del siglo I a.C al final del periodo republicano. a.C conmemora a dos purpurarias:

V(IVUS) D(ECIMUS) VETURIUS D(ECIMI) L(IBERTUS) DIOG(ENES) / (OBITUS) D(ECIMUS) D(ECIMI) L(IBERTUS) NICEPOR / V(IVA) VETURIA D(ECIMI) L(IBERTA) FLORA/ DE SUA PECUNIA FACIUND(UM) COIR(AVERUNT) / SIBI ET PATRONO ET CONLIBERT(IS) / ET LIBERTO / NICEPOR(US) CONLIBERTUS / VIXIT MECUM ANNOS XX / PURPURARIA MARIANEIS / VIV(US) D(ECIMUS) VETURIUS D(ECIMI) I(MULIERIS) L(IBERTUS) PHILARCUR(US)

Esta inscripción dedicada por una mujer llamada Veturia Fedra recuerda a su patrono, Decimus Veturius Diogenes (el cognomen Diógenes indica su origen griego) y su marido y coliberto D. Veturio Nicepor y a otro liberto Philargyro.

Esta mujer pertenece a una familia libertos de la Gens Veturia dedicados al negocio de tintorerías purpurarias. Varias mujeres de esta familia como Veturia Atica y Marianeis, citadas en la inscripción, son purpurarias e intervienen y participaron directamente en el comercio del tinte (JEFFERSON LOANE, H. 1990, 75-77; FERNANDEZ URIEL, 2010, 185-186).

Otras purpurarías fueron Muronia Prima, al menos esposa de un purpurario,(CIL XI, 6604) y Baebia Veneria, cuya condición jurídica no se especifica, tal vez fuera liberta y era trabajadora en la factoría de púrpura constatada en Gades (Bernal et alii...)

D (IIS) M(ANIBUS) S(ACRUM) B<A $>$ EBIA VENERIA $/<\ldots$ PUR $>$ P $<$ U $>$ RARIA / $<$ A(ARA $>$ S(UIS) AVO DULCIS /ANN(ORUM) XXV /B $<$ A $>$ EBIUS/ VENERIOSUS / $<$ A $>$ NNI I M (ENSIUM) III V(OBIS) T(ERRA) $<$ L(EVIS) $>$ (CIL, 1743). 
Singulares son las referencias bíblicas que se encuentran en un conocido pasaje del Nuevo Testamento que se inserta en uno de los viajes de Pablo de Tarso (45-58 d.C) y narra una conversación con unas mujeres que habían acudido a un lugar de oración no muy lejos de Filipos.

Entre estas mujeres se encuentra, Lidia. Cuando conoció a Pablo de Tarso en uno de sus viajes, ella y toda su familia se convierten al cristianismo.

Et quaedam mulier nomine Lydia purpuraria civitatis Thyatirenorum colens Deum audivit cuius Dominus aperuit corintendere his quae dicebantur a Paulo (Hechos Apost, 16, 14).

Desde entonces, pone a disposición de la comunidad cristiana su casa, que se convirtió en una primera iglesia que dirigió hasta su muerte. Lidia aparece de nuevo citada por Lucas (Hechos Apost. 16, 40), donde queda manifiesta su colaboración con las comunidades cristianas. Son pocos los caracteres que se ofrecen sobre esta dama del Nuevo Testamento, pero muy significativos: Es una mujer de origen griego, comerciante de púrpura, de elevado nivel económico, que vivía en Tiro.

\section{MUJERES COMERCIANTES. VENDITRICES}

Estas mujeres «empresarias» o negotitatrices la «banquera «Cesenia, la negotriatrix frumentaria Abundia Megiste, la latifundista Valeria Maxima, la propietaria Julia Felix, tiene en común una buena posición social y disposición de medios bien heredados, buen adquiridos, y socialmente son mujeres respetadas (LAURENCE, R., 1994).

Estas damas no dejan de ser una excepción. La gran mayoría de las mujeres trabajaron en ciertos oficios o regentaron negocios mucho más modestos en pequeños locales, talleres que muchas veces consistían en simples puestos en las calles de cualquier ciudad romana, a las que podríamos denominar «Venditrices».

Algunos de estos oficios o las propias mercancías con las que traficaban son un tanto insólitos como el que ejerce Aurelia Vernilla que era plumbaria, su inscripción funeraria cuenta que regentaba un taller de plomo de forma independiente:

D.M. AVR VERNILLA PLVMBARIA SIBI/ET AVR LVCIO MARITO SVO ET AVR STER/ CORIAE FILIAEPOSVIT QVOD SI QVI SVPEPER/ HEC CORPORA POSVERIT/INFERET R P S C (CIL III, 2117).

Cornelia Venusta, también liberta, era clavaria, trabajaba junto a su marido (clavarius)con probabilidad únicamente se dedicaba a su venta y no a su fabricación.

V F CORNELIA / VENVSTA/ CLAVARIA SIBI ET/ P AEBVTIO M F STEL/CLAVARIO AVG VIR/ ET CRESCENTI LIBERTAE ET/ MVRONI DELICATAE (CIL V, $7023=$ ILS 7636). 
Laturnia Januaria era calcaria en Pompeya, de la que únicamente conocemos su dedicación y la edad de su fallecimiento.

LATVRNIA IANVARIA CALCARIA VIX ANXXXXIV (ILS 7663).

Otra labor que parece impropia del sexo femenino es la que ejercían las bratteariae, Septicia Rufa y Fulvia Melema, dedicadas a la producción de hojas de metal con sus respectivos maridos Septicio Apolonio y Fulcinio Hermero, circunstancia que hace suponer a Le Gall que se dedicarían únicamente a vender las hojas que fabricaban sus según se desprende de la lectura de las siguientes inscripciones (LE GALL, J.,1969):

A SEPTICIVS A L/ APOLLONIVS/ BRATTIARI/ SEPTICIA A L/ RVFA BRATTIA/ OLLAS II (CILVI, 6939).

C FVLCINIVS C L/HERMEROS/ BRATTIARIVS/ FVLVIA MELEMA/ VIXIT ANNIS XXXXVIII/ BRATTIARIA (CIL VI, 9211).

Aún es más sorprendente que una mujer se dedicase al negocio armamentístico, pero en las Notitia Dignitatum y en particular, entre los Insignia viri illustris magistri officiorum, hay referencia a dos sagittariae -fabricantes de flechas- una en la provincia Itálica y otra en la Galia, y una ballistaria -fabricante de ballestas- también gala.

Hay una segunda referencia a unas scutariae et armamentariae de Damasco, Antioquía y Edesa.

Quizá en el ámbito del negocio bélico puedan llegar a incluirse a las denominadas coronariae, dedicadas a ensamblar coronas y guirnaldas, que con probabilidad lucirían los militares héroes de campañas a la hora de celebrar sus triunfos así como, en diferente contexto, los vencedores de juegos y competiciones deportivas.

Estos negocios fueron una excepción. La mujer se dedicó a oficios y especulación de objetos de lujo, perfumes, alimentos o distintos ámbitos dentro de la producción textil.

Destaca la dedicación de la mujer a la fabricación y venta de perfumes, una profesión relativamente bien atestiguada, que vendían e incluso fabricaban, pues los epitafios no clarifican si estas mujeres ungüentarias eran ellas mismas quienes fabricaban dichas sustancias (en griego Myropolides) o sólo se dedicaban a su venta y distribución. Aunque los filósofos se opusieron a los perfumes que consideraron un lujo engañoso, debía ser un negocio rentable y sin duda tuvo un excelente mercado. En Roma están atestiguadas 19 perfumistas. Licinia Primigenia era ungüentaria y su hijo Licinio Amomo le dedicó un epitafio alabándola como «respetabile matri b(ene) m(eritis)».

D M/ LICINIAE PRIMIGENIAE/ VVNGVENTARIAE/ LIC AMOMVS F MATRI B M/ VIX A LXXI (CIL X, 1965). 
Otra ungüentaria Llamada Biena Cora es conmemorada por su marido.

BIENA COR/ VNGVENTARIA AB D/ NON HIC OLLA MEOS CINERES A V/SET PASSIM MATER TERRA TEGIT/ CONIVGIS HOC FECIT CARIMIH/ PRAESARIT IVNCTAE VIVA QVOT ANT/ CVVM QVOTRIGINTA ANNIS SINI IAB/ IN CVIVS MANIBVS (CIL VI, 10006).

Un negocio próspero era la venta de incienso (Thuris),en el que destacó al parecer en Roma la familia de los Trebonii cuyas inscripciones incluyen a mujeres (cinco hombres y dos mujeres Thuraii) (KORPELA, J, 1995).

Había establecimientos que vendían prácticamente todos estos productos: perfumes, ungüentos, incienso, plata, gemas y joyas, pero, por lo general la joyería se encuentra como un mercado muy especializado, del que citaremos a Babbia Asia, gemmariaque tenían sus negocios en la Via Sacra de Roma. Su nombre denota un origen oriental.

Sus dos libertos ensalzan su conocimiento en el diseño y técnica de fabricar joyas, su habilidad en la incisión de piedras preciosas, en fijar y enjertar perlas «donde sus dedos son diestros y agiles y demuestran talento como vendedora».

La joyería, a su vez, contaba con verdaderas especializaciones como Domicia que comercia solo con perlas (margaritaria). No puede afirmarse que exclusivamente se dedicara a la compraventa, sino que posiblemente también las cultivara:

DOMITIA C N L/ MARGARITARIA/ MV S/ V A. (CIL VI, 5972, Igualmente, en CIL VI, 9435).

En los mercados (nundinae), muchos puestos de artículos de alimentación estuvieron regentados por mujeres. Algunas inscripciones funerarias están dedicadas a conditariae, o asentadoras de alimentos, como las Fabariae Antistia Victorina, que ejercía su oficio junto con su marido (CIL VI, 9277), o la de Glyco (CIL IV, 4001) 6 .

V...AE.../ GENIVM COL/ ANTISTIA VICTORINA/ FABARIA/ PRO SALVTE SVA/ ET C ANTISTI ELAINI/ ET VICTORINI ET SALVI/ ET HORATIONIS ET CARAE/ SII V S. (CIL III, 153)

O la pescadera (piscatrix) Aurelia:

AVRELIA C L NAIS/ PISCATRIX DEHORREIS GALBAE/C AVRELIVA C LA PHILEROS/ PATRONVS/ L VALERIVS L. L. SECVNDVS. (CIL VI, $9801=I L S$ 7500)

En la casa de Julia Felix, en Pompeya hay una pintura que representa una joven vendedora de frutas (pomararia) y en las fuentes literarias, una vendedora de verduras (Holicaria) es citada por Petronio (SAMPAOLO, V. 1991).

6 Otras vendedoras de habas y legumbres en CIL, III, 153 y 6672. 
[...] Itaque quocumque ieram, eodem revertabar, donec et cursu fatigatus et sudore iam madens accedo aniculam quandam, quae agreste holus vendebat [...]. Sat., 6, 4: [VI]

Otras fueron panaderas (furnaria) (CIL VIII, 24678), vendedoras de carnes (porcinaria) (CIL; V, 8706), de trigo y cebada (Frumentariae), legumbres (legumenariae). Si salimos de Roma, encontramos otras vendedoras como la vendedora de semillas en Preneste, la de habas en Beirut, A veces eran gentes con cierta solvencia ya que tenían varios libertos y esclavos a su servicio.

\section{LA INDUSTRIA TEXTIL}

La franja entre la mujer comerciante y la obrera y la sierva contempla una serie de actividades donde se situaría a la mayoría de las mujeres trabajadoras, su trabajo es más humilde y su situación social económica más precaria y sumida en el anonimato.

Una gran parte queda restringida al ámbito doméstico, a los servicios y en gran medida, a manufactura textil de lo que hay una gran variedad y, posiblemente, donde se encuentra la mayor cantidad de información.

Por lo general se compraban los paños y tejidos de los lugares donde se producían a través de vendedores especializados.

La gente rica tenía sus propios obreros y personal apropiado a su servicio para la confección de ropa en su propia casa que eran servidores domésticos de ambos sexos, pero casi siempre mujeres, que recibían diversos nombres (fertificii; festificus /vestifex/vesticinae). La gente común solía utilizar la ropa ya confeccionada.

Como se advierte en las fuentes, el trabajo textil de las mujeres no quedó reducido al ámbito exclusivamente doméstico y algunas mujeres lograron montar su propio negocio.Susan Treggiari ha contabilizado siete en Roma y dos en la península Itálica que se atestiguan como profesionales con un negocio de un cierto nivel (TREGGIARI, S., 1976).

Martia Prima, de origen galo, logró comprar su libertad, ganarse la vida e incluso establecer su propio negocio de manufactura textil (vestiaria). Murió a los 46 años, según reza la inscripción que le dedica otro liberto Tito Thoranio Salvio (liberto de Tito).

Una situación semejante parece tener las vestificae Pompeya Trofime y Agrippina dedicadas a la fabricación de vestidos:

D M/ PONPEIAE TROFHIME/ VESTIARIA RVFINA/ FILIA MATRI/ B M F VXOR/ NARCISIONIS/ CONDITOR/ GREGIS RYSSATAE (CIL VI, 10072 = ILS 5305)

HELICONIS NARCISSI/ AVGVSTIANI ET HELICONDIS/ FILIA VIX ANN III/ HELICONIS AGRIPPINAE/ VESTIFICA VIX ANN XXVI/ POST TRIENNIVM DECESSIT EADEM/ DIE QVA FILIA

(CIL VI, 5206d) 
En Hispania Valeria Sevenina, dama de considerable riqueza y prestigio social, era patrona de un collegium y propietaria de talleres textiles (CIL, II, 5825; GALLEGO FRANCO, H., 1993,117).

Otra inscripción cita a una vestiaria dentro de un grupo de cinco libertos de Cermalo Minusculo que conmemoran a su patrón, también liberto (CIL VI, 33922 = ILS 7570).

Existían talleres dedicados a arreglos y zurcidos de ropa (sarcinatrices) algunos de forma autónoma.

En este mismo ámbito textil, y en cuanto a la confección de ropajes, llama la atención la Sellia Epyre, esposa de un ciudadano romano, Q. Futius Olimpicus, cuyas cenizas se han reunido en la misma urna funeraria, quienes se dedicaban a un comercio de lujo que consistía en vender vestidos bordados en oro (aurivetrix).

SELLIA/ EPYRE/ DE SACRA VIA/ AVRI VESTRIX/ Q FVTI OLYMPICI (CIL VI, 9214 = ILS 7692).

Viccentia era una niña trabajadora, una hilandera de oro que solo tiene nueve años, a la que recuerdan sus padres, de los que no conocemos su ocupación,posiblemente de condición humilde.

VICCENTIA DVLCISSIMA/ FILIA/ AVRINETRIX QAE/ VIXIT AN VIIII M VIIII (CIL VI, 9213).

Otro oficio dentro del ámbito textil es trabajar con lana (Lanifacium) que podía tener distintos matices, significación y términos.

El trabajo de hilado fue considerado una constante femenina, personificada en los vocablos lanifica, quasillaria, lintearias, sobre todo el primero, que en su forma adjetivada califica, no tanto un oficio, sino una de las virtudes de la matrona romana alusivo a la honradez y la pulcra vida familiar de una esposa.

Cuando se trata de un oficio o un trabajo, eso sí, propio de la mujer, era realizado por siervas, obreras y esclavas, mujeres de muy baja capacidad económica. La mayoría de las tejedoras, zurcidoras, hilanderas etc. debían de ser trabajadoras, obreras empleadas al servicio de importantes talleres regentados por negotiatores de este tipo de empresa o, simplemente domésticas, aunque podían ejercer como independientes, tal y como se desprende del sombrío pasaje de Apuleyo (Met., 9, 5-6), donde nos narra el caso de una mujer pobre, hilandera, que consiguió mantener a su familia como podía mientras su marido, trabajador en fabriles operae, obligado por el officinatora realizar «vacaciones forzosas».

Estas hilanderas tiene una extraña distribución en Roma, hay 11 conocidas, ocho concentradas en un lugar determinado, en el columbario de los Statilii, parecen de una familia de ricos tejedores.

* Otra situación era la de las lanipendae o -vendedoras de lana-. El trabajo textil de estas comerciantes de lana no es hilarla, sino venderla, a lo que se dedicaban Lucilia Benigna, citada junto a un argentario y Julia Soteridia: 

3157)

LVCILIA[/ BENIGNA[/ LANIPEND/ VIVA SIBI ET VRB[/ARGENTARIO (CIL IX,

Iulia Soteridia se dedicaba a la venta de la lana elaborada, incluso confeccionaba vestidos con la lana como materia prima, en un taller propiedad de su marido, Julio Primo donde cuatro esclavas o libertas ayudaba a esta vendedora:

D M/ IVLIAE SOTERIDI/ LANIPENDAE V A LXXX/ FECERVNT/ M IVIVS PRIMVS/ IVLIA MVSA IVLIA THISBE/ IVLIA AMPLIATA/ IVLIA ROMAN (CIL VI, 9498).

Con relación al trabajo de hilado, no de lana, sino de lino, hallamos la sintética inscripción de una lintearia de Tarraco, tan escueta que no da pie a conclusión cierta:

FVLVIA LINTEARIA. (CIL II, 4318aㅗ

Sin salir en el ámbito de la indumentaria, hallamos mujeres dedicadas al comercio o fabricación de calzado, en concreto, Septimia Stratonice, una sutrix independiente de Ostia. Parece ser que se trata de una ingenua conmemorada por su esposo e hijo (TREGGIARI, S., 1976: 66-79).

HOC SEPVLCRVM/ M ACILIVS IS [/ CESSIT DONATIONE/ SEPTIMIAE STRATONICE/ SVTRICI/AMICE CARISSIME/ OB BENE

FACTA/ AB EA IN SE DIMIDIA/ PARTE ET [] ACILIO/FORTVNATIANO/ FILIO] (CIL 14 Suppl. 4698.).

\section{MUNERI ET OFFICIAE MULIERIS}

En las calles de cualquier ciudad romana se situaban mujeres de distintos oficios que ofrecían sus servicios.

Era muy frecuente hallar a peluqueras (ornatrices). Era un trabajo que se podía ejercer en casa de los ricos pero también de forma independiente con frecuencia e, incluso ejercían de barberas tonstrix como comenta Marcial:

"Una peluquera se haya sentada al comienzo de la calle Subura donde cuelgan los vergajos sangrantes de los esbirros y en donde muchos zapateros están al frente por frente del barrio de los alfareros. Pero esta peluquera, Amiano no afeita, te digo-¿Pues qué es lo que hace? Desuella» (MARCIAL II, XVII) ${ }^{7}$.

Hay varias peluqueras documentadas en inscripciones funerarias de Roma como Nostia Dafne que tenía su establecimiento en el Vicus Longus, que se re-

7 También en PLAUTO, Truc., 4, 3, 791; BLÁZQUEZ, J. M., 2004, 57-66 
cuerda junto con un artesano de oro -aurifex- de la misma calle, con probabilidad su marido.

NOSTIA L/ DAPHNE/ ORNATRIX DE/ VICO LONGO/ M NERVIS M L/ QVADRATO AVRIFEX DE/ VICO LONGO (CIL VI, 37469 = ILS. 9426).

Esta inscripción parece vinculada otra Cleopatra de la misma calle:

NOSTIA/ IS DAPHINIDIS L/ E CLEOPATRA/ ORNATIX DE VICO LONGO (CIL VI, $9736=$ ILS. 7618)

Al hallarse esta última incompleta, no podemos saber con certeza si Cleopatra -que es una peluquera de la misma calle- puede relacionarse con la Nostia Daphne de la inscripción anterior.

También era ornatrix Pollia, una liberta de otra mujer, Urbana, que trabajaba como peluquera, conmemorada por otro liberto de otra gens, probablemente su marido, que es barbero en el mismo establecimiento. Otra más trabaja en el campo de Marte conmemorada posiblemente por su marido, tal vez peluquero, tal vez barbero.

POLLIA C [MVLIERIS] L/ VRBANA ORNAT DE/ AEMILIANIS OLLAS II/ M CALIDIVS M L TOSOR/ APOLONI DE AEMILIANIS (CIL VI, 37811 = ILS. 9427).

Por último, algo curioso, Iulia Agele, es una resinaria, una depiladora que utiliza resina.

D M/ IVILAE AGELE RESINARIAE/ QVAE VIX AN LXXX/ IVLIA IRENE PATRONAE/ B M F ET SIBI ET SVIS/ (CIL, VI, 9855 = ILS7658).

Otras mujeres, muchas anónimas que también solían ofrecer sus servicios en las casas y en las calles, como adivinas, parteras, curanderas, actrices, mimas, pantominas...8.

\section{MUJERES EN LOS ESTABLECIMIENTOS TABERNARIAE Y STABULARIAE}

Las fuentes literarias demuestran que eran en su mayoría mujeres quienes atendían y regentaban los establecimientos dedicados a servicios de hospedería, comidas y bebidas y los burdeles prostíbulos (deversorius, hospitium,stabulum, caupo) que debían ser muy numerosos.

8 Es escasísima la documentación sobre estas mujeres que trabajaban en las calle, por lo que es fundamental la información que proporcionan las fuentes iconográficas, Es notable el trabajo de PEREA YÉBENES, S, 2004, 11-43. Sobre la actividad de la mujer en la medicina ver entre otros: BLIQUEZ, L. J., 1995; DIMOPOULOU, A, 1999; GOUREVITCH, D., 1984 y 1996. 
Los nombres Stabulum y Prostibulum tienen un origen etimológico del término latino, prostituere, que significa «exhibir para la venta», nos da una idea del carácter de esta actividad, en la que la mujer se pone literalmente «a la venta». Aunque, "prostíbulo», puede considerarse como un nombre procedente de los lugares donde solían colocarse para atraer a los clientes, que era «delante de» las «stabula», que aunque su significado más habitual es: cuadra, establo, la palabra «stabularia» significa «posada», y esta era una localización muy habitual (DE FELICE, J., 2001).

Hay nombres de hombres pero al parecer no eran pocas las mujeres propietarias de estos establecimientos como demuestran las fuentes epigráficas que citan en femenino a hospitae, popinaria, caupona como el de Amemone, que tenía un establecimiento en Tibur:

DVLCIS/ LATET HOC AMEMONE SEPVLCRO/ FAMA VLTRA FINES PATRIAE POPINARIA NOT (CIL VI, 1819).

Recordemos el caso de la ya citada Calpurnia, vinaria de Pompeya: teóricamente puede ser una comerciante de vino, pero también, con mayor probabilidad, puede tratarse de una mujer que regentaba una taberna:

SVAVIS VINARIA/ SITIT ROGO VOS ET/ VALDE SITIT CALPVRNIA TIBI DICIT VAL (CIL XIV, $3709=$ ILS. 7477)

Sin embargo, ambas son una excepción. Parece ser que las hosteleras y taberneras no tenían muy buena reputación. No es fácil encontrar nombres de estas mujeres porque estaban consideradas como meretrices Las chicas encargadas de las Cauponiae (copae) tenían una pésima fama, por lo que no debe sorprendernos su anonimato, pues no parece muy afortunado que figurara su nombre junto a esta clase terminología correspondiente en la dedicación de una lápida o en un epitafio (FORBIS, E. P, 1990).

Las fuentes jurídicas (ULP. Digesto 23, 2, 43pr y 9), nos aclara que la prostituta pública no es tan sólo la que vive en un burdel, sino también la que no se recata en un local hostelero lo que demuestra que en estos locales había esclavas prostitutas, hecho que parece confirmar las palabras de Apuleyo (Met, I, 8, 75)donde se refiere a una hostelera que posiblemente se dedique a la prostitución, de igual forma, el texto de Petronio (Sat., 6, 776), donde concretamente cita a las meretrices como dueñas de burdeles.

Es enormemente elocuente el diálogo que se encuentra grabado en el campo epigráfico superior de la estela romana de Aesernia, ahora en el Museo de Nápoles, en el que el cliente de una posada arregla cuentas con la dueña, en el pago incluye los servicios de una muchacha como algo tan normal como podía serlo la comida, el alojamiento o la paja para la caballería, ambos representados en la parte inferior de la estela: 


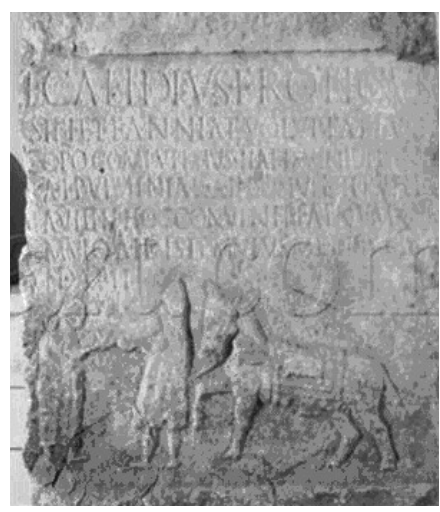

1) Estela de Aesernia, Museo de Nápoles.

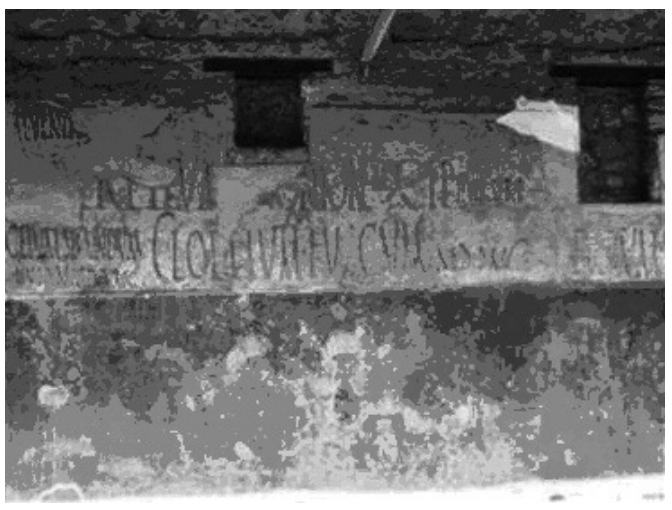

2) Grafittidel Termopolio de Asellina (Pompeya).

Posadera, hágame la cuenta.

—Un sextario de vino. Por el pan un as; por el pulmentarium3, dos ases.

-Conforme.

-Por la muchacha, ocho ases.

-De acuerdo también.

- Heno para la mula, dos ases.

-Bien.

Pero si contamos con otro tipo de documentación valiosísima y directa: los grafitti pompeyanos, escritos por ellas mismas, por clientes agradecidos o malévolos. Los nombres de las camareras y las prostitutas se encuentran garabateados en las paredes de Pompeya, incluso de carácter electoral, apoyando o criticando a algún candidato Pocas mujeres respetables escribieron esta clase de graffiti, pero las que se mezclaban con las multitudes -las camareras y prostitutas- son responsables de muchas pintadas de esta clase (que incidentalmente indicaban que sabían escribir): por ejemplo, «Sucula te pide que hagas edil a Marco Cerrinio». Otro ejemplo de la intervención de estas mujeres en las «campaña electorales» son los graffiti de las paredes del termopolio de Asellina y las chicas que atendían a la clientela Aegle, Muría y Smyrina, en la Via de la Abundancia pompeyana (CIL, IV, 7863) ${ }^{9}$.

Como conclusión; Hay varios tipos y escala de mujeres

Dentro de las negotiatrices:

- Alta escala social y económica pueden ser catalogadas de comerciantes y empresarias de alto estatus no sólo por su situación (Patriciado) sino además

9 Existe sobre ello una gran cantidad de estudios, Ver entre otros: CANTARELLA; E., 1999; SAVUNEN, L.,1995; FORBIS, E. 1990. 
se presume una gran capacidad económica, y su implicación en el mundo mercantil podía producirse de forma directa e indirecta, por ejemplo, a través de su actividad financiera como meras inversoras y por medio de la figura del praepositus, Son las mujeres dedicadas a la navegación, así como las que regentaban grandes empresas y las importantes propietarias territoriales y de inmuebles (SETÄLÄ P. et alii, 2002).

- A la pequeña empresa y a actividades comerciales de índole menor y al pequeño comercio se dedican mujeres de poca capacidad económica

- Son mujeres de cualquier status social, pero la mayoría son libertas sin que para ello influya en su situación económica. Difícilmente una mujer libre se dedicaría a actividad mercantil, ya que ésta puede ser considerada como indigna, no sólo era cuestión de sexo, sino también ética e ideológica, porque no sería muy corriente que una mujer respetable, cuyas circunstancias familiares la obligaran a trabajar, su actividad laboral fuera conocida e incluso, conmemorada. Hay libertas adineradas que formaban parte de una clase burguesa acomodada, posición que les permitía construirse sus propias sepulturas y vestirlas con las correspondientes lápidas y epitafios para ser conmemoradas convenientemente. Estas son precisamente las que manifiestan con orgullo su labor y su trabajo.

Su trayectoria debía ser muy similar: Posiblemente una mujer esclava que conseguía ahorrar suficiente dinero durante su esclavitud para comprar su propia libertad y llegar a convertirse en una mujer libre, pero además tendría que continuar ganándose la vida.

Por los epitafios también se deduce que no era inusual que esta mujer se casara con su patrón, un hombre libre, adquiriendo la libertad y continuando ambos en su trabajo o negocio. El matrimonio, además, ofrecía a esas libertas la posibilidad de escalar en su posición económica y en su consideración social. Sin embargo no era corriente que una mujer libre contrajera matrimonio con su liberto,

En este marco estas mujeres tuvieron un gran papel de apertura y libertad social de las mujeres en el Alto Imperio (TREGGIARI, S., 1993).

Sobre el destino y la vida de las mujeres más pobres, aun siendo libres, me permito expresar las palabras de Pomeroy: «de ellas y su existencia sólo puede suponerse». POMEROY, S, 1987,108).

Finalmente, recordemos el epitafio funerario de una mujer alabada por su virtud y también por su trabajo.

SCANTIAE REDEMPTAE IN/COMPARABILISSIMAE FEMINAE QUE/IUS DE VITAE DOCUMENTA NON SUFFICIT / MEDIOCRITAS HOMINUM AT CUMULUM LAUDIS / PERVENIRE. FUIT NAMQUE IUVENIS ISTA / OMNI GENERE LAUDIS CONDIGNA: PRIMO DEIFICAE / SANCTITATIS PUDICITIAE; VALLATA HONESTATE MORUM / [OR] NATA; PIAETAS (!) IN PARENTIBUS PROCLIVA; CASTITATE INLUSTRIS / [T] ENACITATIS; MAGISTRA VER(E)CUNDIAE; ANTISTIS DISCIPLIN[AE IN] MEDICINA FUIT / UT CONTEMNERET IUVENTUTEM; 
NAM MARITUS AM[AVIT] / COIUGEM FAMILIAREM SALUTIS ET VITAE SUAE NU[TRIC(EM)]. / HAEC VIXIT ANNIS XXII, MENSIBUS X. / FL(AVIUS) TARENTINUS ET SCANTIA REDEMPTA / PARENTES FILIAE DULCISSIMAE / SIBIQUE FECERUNT.

(CIL X, 3980)

Scantia Redempta, mujer incomparable, transmitió un modelo ejemplar de vida ante la mediocridad de los hombres. Mereció y fue digna de toda alabanza: íntegra, pura, supo rodearse de costumbres honestas, piadosa, generosa con los suyos, admirable en su castidad, fue tenaz y discreta. Maestra versada en el arte de la medicina a pesar de su juventud. La amó su marido, que se benefició de su vida fecunda y llena de virtudes. Vivió 22 años y 10 meses. Flavio Tarento y Scantia Redempta, padres de tan dulcísima hija, dedican este monumento. (Traducción propia)

\section{BIBLIOGRAFÍA}

ADCOCK, F. E.: «Women in Roman Life and Letters», Greece \& Rome, Vol. 14, No. 40 (Jan., 1945), 1-11.

- Society and Politics in Ancient Rome, London, 2009.

AA.VV.: Extranjeras en el mundo romano, G. BRAVO CASTAÑEDA y R. GONZÁLEZ SALINERO (eds) Anejos de Gerion, VIII, 2004, P. Universidad Complutense de Madrid.

ANDREAU, J.: Banking and Business in the Roman World. Cambridge and New York: Cambridge University Press, 2001.

ALFARO, C. (ed.), «Dossier: Mas allá de la Labor matronalis. Aspectos del trabajo profesional femenino en el mundo antiguo", Saitabi 49 (1999), 205-359.

BALCH, D. «Rich Pompeiian Houses, Shops for Rent, and the Huge Apartment Building, Herculaneum as Typical Spaces for Pauline House Churches», JSNT 27.1 (2004), 2746.

BLÁZQUEZ, J. M. «Mujeres extranjeras en Roma en la obra de Marcial», Extranjeras en el mundo romano, G. BRAVO CASTAÑEDA y R. GONZÁLEZ SALINERO (eds) Anejos de Gerion, VIII, 2004, 57-66.

BLIQUEZ, L. J. (1995), «Gynecology in Pompeii,» PH. J. VAN DER EIJK- HFJ. HORSTMANNSHOFF - PH. SCHRIJVERS (eds.), Ancient Medicine in its Socio-cultural Context, Amsterdam, 1995, 209-223.

BOVE, L.: Processuali dalle tabulae pompeianae de Murécine Naples, 1979 BUONOPANE, A; CENERINI, F. (eds.), Donna e lavoron el la documentazione epigrafica. Atti del I Seminario sulla condizione femminil en ella documentazione epigrafica, Faenza, Fratelli Lega Editori, 2003.

CAMODECA, G.: Tabulae. Pompeianae Sulpiciorum Rome, 1999.

CANTARELLA, E.: La mujer romana, Santiago de Compostela, 1991, p. 96.

— «Qualche considerazione sul lavoro femminile a Pompei», Saitabi 49 (1999), 259- 272.

CASTILLO, A. del «El papel económico de las mujeres en el alto imperio romano», Revista Internacional de Sociología, 32 (1974), pp. 59-76.

- «Legislación romana y liberación femenina: una relación inconsciente», Lvcentvm, VII-VIII, (1988-89), 161-169.

CID LÓPEZ, R. M.: «La maternidad y la figura de la madre en la Roma antigua», Nuevas visiones de la maternidad, A. I. BLANCO GARCÍA, BLANCA DOMENECH DELGADO, M. S. LÓPEZ RODRÍGUEZ, R. MARCOS SANTIAGO (eds.), 2002, 11-50.

— «La educación de la niña romana: de puella a matrona docta», Bien enseñada: la forma- 
ción femenina en Roma y el occidente romanizado, R. FRANCIA SOMALO, V. ALFARO BECH (eds.) 2001, 19-44.

COOLEY, A. E. COOLEY M. G. L.: Pompeii: A Sourcebook. London/New York, Routledge, 2004.

D'ARMS, J. H., «Memory, Money, and Status at Misenum: Three New Inscriptions from the Collegium of the Augustales", JRS 90 (2000), 126-44.

D'AVINO, M., La donna a Pompei, Nàpoles, 1964.

DE FELICE, J., Roman Hospitality: the Professional Women of Pompeii (Warren Center PA: Shangri-La), 2001.

DIMOPOULOU, A: «Medica, obstetrix, nutrix: les femmes dans les métiers médicaux et paramédicaux dans l'antiquité grecque et romaine», Saitabi 49 (1999), 273-287.

EICHENAUER, M.: Untersuchungenzur Arbeits welt der Frau in der römischen Antike, Frankfurt, Peter Lang Verlag, 1988.

FORBIS, E.P: «Women's Public Image in Italian Honorary Inscriptions», American Journal of Philology, 111, 1990, 493-512.

FRENCH, V.: «Midwives and Maternity Care in the Roman World», Helios 12 (1986/2), 6984.

GARCIA GARRIDO, M. J., El comercio, los negocios y las finanzas en el Mundo Romano, Madrid, 2001.

GOUREVITCH, D.: Le Mal d'être femme: la femme et la médecine dans la Rome antique, París, 1984.

— «La gynécologie et l'obstétrique», ANRW II, (1996), 37.3, 2083-2146.

GUIRAL, C.; JIMÉNEZ, J. L.; MAR, R.: «Misión arqueológica española en Pompeya. Informe de los trabajos realizados durante las campañas de 1989-1990 en la casa-caupona (1, 8, 8-9) de L. Vetutius Placidus", AEspA 64 (1991), 293-317, Abb.

JONGMAN, W., The Economy and Society of Pompeii [Eumachia's building]. Amsterdam: Gieben, 1991, 179-84.

KAMPEN, N., Image and Status: Roman Working Women in Ostia, Berlín, Gebr. Mann Verlag, 1981.

LAURENCE, R., Roman Pompeii: Space and Society London, 1994.

LÁZARO GUILLAMÓN, C.: «Mujer, comercio y empresa en algunas fuentes jurídicas, literarias y epigráficas», RIDA (2003), 155-193.

LE GALL., J. «Métiers de femmes au Corpus Inscriptionum Latinarum», REL 47 bis (1969), 123-130.

MAIURI, A., «Duelscrizioni Veneree Pompeiane», Saggi di varia antichità, Venice, 1954, 284302.

- Herculaneum, 7th English edition, Rome, Libreria dello Stato, 1978.

- Pompeii. 15th ed. Rome: Libreria dello Stato, 1978.

— «Guilia Felice, gentil donna pompeiana», Pompeii ed Ercolanofra case e abitanti, Milan, 1964, reprint, 1983). 75-84.

MAURIN, J.: «Labor matronalis: aspects du travail féminin à Rome», a: E. Lévy (éd.), La femme dans les sociétés antiques. Actes des colloques de Strasbourg (mai 1980 et mars 1981), Estrasburg, AECR, 1983.

MONTERO, S., Diosas y adivinas. Mujer y adivinación en la Roma antigua, Madrid, 1994.

- «Mujeres extranjeras en la obra de Valerio Máximo», Extranjeras en el mundo romano, G. BRAVO CASTAÑEDA y R. GONZÁLEZ SALINERO (eds) Anejos de Gerion, VIII, 2004, 45-56.

MORRETTA, S.: «Donne imprenditrici nella produzione en el comercio dell'olio betico» (I-III sec. D.C.), Saitabi 49 (1999), 229-245.

MUSTAKALLIO, K. «Legendary Women and Female Groups in Livy», Female Networks and the Public Sphere in Roman Society, Roma, 1999, 53-64.

PARSLOW, Ch. C., The Praedia Iuliae Felicis in Pompeii (Italy), Ph.D. dissertation, Duke University, 1989. 
- Rediscovering Antiquity: Karl Weber and the Excavation of Herculaneum, Pompeii, and Stabiae, Cambridge: Cambridge University Press, 1995.

PEPPE, L., Posizione giuridica e ruolo sociale della donna romana in età repubblicana, Milano, 1984.

PEREA YÉBENES, S.: «Mujeres extranjeras en Roma y en cualquier lugar: mujeres mimas y pantomimas, el teatro en la calle y la fiesta de Flora», Extranjeras en el mundo romano, G. BRAVO CASTAÑEDA y R. GONZÁLEZ SALINERO (eds.) Anejos de Gerion, VIII, 2004, 11-43.

POMEROY, S.: Diosas, rameras, esposas y esclavas: mujeres en la antigüedad clásica, Madrid, 1987.

RAWSON, B. «Roman Concubinage and Other De Facto Marriages». TAPA 104, (1974), 279-305.

RICHARDSON, L. Jr., Pompeii: An Architectural History. Baltimore: Johns Hopkins, University Press, 1988.

SALLER, R.: «Pater Familias, Mater Familias and the Gerended Semanties of Roman», Classical Philology, 94, (1999), 84-87.

SAMPAOLO, V., «Villa di Giulia Felice», Pompeii: Pitture e Mosaici, ed. Ida Baldassarre et al. Milan: Istitutodella Enciclopedia Italiana. Vol. 3, (1991),184-310.

SAVUNEN, L. (1995), «Women and elections in Pompeii», in R. Hawley \& B. Levick (eds.), Women in Antiquity: New Assessments (Routledge: London), pp.194-206.

SETÄLÄ P. et alii, «Female Networks and Public Sphere in Roman Society», Acta Instituti Romani Finlandiae, 22, 1999.

- «Women, Wealth and Power in the Roman Empire», Acta Instituti Romani Finlandiae 25, 2002.

TREGGIARI, S., «Jobs for Women», AJAH 1, (1976), 76-104.

- Roman Marriage: Iusti Coniuges from the Time of Cicero to the Time of Ulpian, Oxford and New York: Clarendon Press, 1993.

WALLACE-HADRILL, A. «Imaginary feasts: pictures of success on the Bay of Naples», JRA Supp. 57 (2004), 109-26. 
